\title{
CASIM Input Parameters for Various Materials
}

\author{
A. J. Malensek and A. J. Elwyn \\ Fermi National Accelerator Laboratory \\ P.O. Box 500, Batavia, Illinois 60510
}

FERMILAB-TM-1898

P.O. Box 500, Batavia, Illinois 60510

July 1994 


\section{Disclaimer}

This report was prepared as an account of work sponsored by an agency of the United States Government. Neither the United States Government nor any agency thereof, nor any of their employees, makes any warranty, express or implied, or assumes any legal liability or responsibility for the accuracy, completeness, or usefulness of any information, apparatus, product, or process disclosed, or represents that its use would not infringe privately owned rights. Reference herein to any specific commercial product, process, or service by trade name, trademark, manufacturer, or otherwise, does not necessarily constitute or imply its endorsement, reconmendation, or favoring by the United States Government or any agency thereof. The views and opinions of authors expressed herein do not necessarily state or reflect those of the United States Government or any agency thereof. 


\title{
CASIM Input Parameters for Various Materials
}

\author{
A. J. Malensek and A. J. Elwyn
}

14 July 1994

\section{Introduction:}

During the past year, the computer program CASIM [1] has been placed in a common area from which copies can be obtained by a wide array of users. The impetus for this arrangement was the need to have a standard code that could be maintained and transported to other platforms. In addition, an historical record would be kept of each version as the program evolved.

CASIM requires a series of parameters (input by the user) that describe the medium in which the cascade develops. Presently a total of 9 materials can be defined. Occasions arise when one needs to know the properties of materials (elements, compounds, and mixtures) that have not been defined. Because it is desirable to have a uniform set of values for all CASIM users, this note presents a methodology for obtaining the input parameters for an arbitrary material. The parameter names and units are listed in TABLE 1. They are read in by the Subroutine CASIM_PROG from the user supplied file CASIM.DAT.

TABLE 1

\begin{tabular}{|l|l|l|}
\hline Parameter & Name & Unit \\
\hline Atomic Number & ZT & -- \\
\hline Atomic Weight & AT & amu \\
\hline Density & RHO & $\mathrm{gm} / \mathrm{cm}^{3}$ \\
\hline Ionization Potential & VIP & electron volts \\
\hline Radiation Length & RALG & $\mathrm{cm}$ \\
\hline Nuclear Radius & RFM & fermi \\
\hline Elastic Cross Section & SIGL & barn \\
\hline
\end{tabular}




\section{Material Parameters:}

In typical cases, the density $\rho$ and chemical composition $\mathrm{C}_{\mathbf{i}} \mathrm{D}_{\mathbf{j}} \mathrm{E}_{\mathbf{k}}$ (where the capital letters represent dummy element symbols) of the material are known. For mixtures the fraction by weight $\mathbf{w}_{\mathrm{m}}$ of each of the constituent elements $m$ is also known. Using $\rho, C_{i} D_{j} E_{k}, w_{m}$ and $m$, one can obtain a set of input parameters for an arbitrary material.

(a) Atomic Weight and Atomic Number--AT and ZT.

For an element, $\quad \mathrm{AT}=\mathrm{A}$

$$
\mathrm{ZT}=\mathrm{Z}
$$

For a compound or mixture containing $\mathrm{n}$ constituent elements,

$$
\begin{array}{r}
A T=\sum_{m=1}^{n} w_{m} * A(m) \\
Z T=\sum_{m=1}^{n} w_{m} * Z(m)
\end{array}
$$

Note that for a compound such as $\mathrm{C}_{\mathbf{i}} \mathrm{D}_{\mathbf{j}} \mathrm{E}_{\mathbf{k}}$,

$$
w_{m}=w_{c}=\frac{i * A T(C)}{i * A T(C)+j^{* A T}(D)+k^{*} A T(E)}
$$

(b) Ionization Potential, Radiation Length, Nuclear Radius and Elastic Cross Section--VIP, RALG, RFM, and SIGL.

All of these are obtained as functions of AT and ZT.

The semi-empirical formula given by Segre[2] is used for the ionization potential,

$$
V I P=9.1 * Z T\left(1 .+1.9 *(Z T)^{-2 / 3}\right)
$$

This representation is plotted along with the data from the Radiological Health Handbook[3] in Figure 1. 
The radiation length is calculated from a formula in the Particle Properties Data Booklet[4] that approximates the values tabulated by Tsai[5].

$$
\begin{aligned}
& X_{0}\left(g / \mathrm{cm}^{2}\right)=\frac{716.4 * A T}{Z T *(Z T+1) * \ln (287 . / \sqrt{Z T})} \\
& \operatorname{RALG}=X_{0} / \rho
\end{aligned}
$$

Nuclear radii as measured in proton-nucleus scattering at $20 \mathrm{GeV}$ by Bellettini et al.[6], are compared with those determined in electronnucleus scattering [7] in Figure 2. As pointed out in ref.[6], agreement is quite good for the lighter elements, but radii obtained in proton scattering are larger by about 0.5 fermi for the heaviest nuclei. For AT between 6 and 12 the nuclear radius is approximated by a constant. For masses of 12 and greater, the solid line is a fit of the Bellettini (proton) data to the customary relation $R F M=r_{0} A^{1 / 3}$.

$$
\begin{aligned}
& \boldsymbol{R} \boldsymbol{F M}=3.4 \\
& 6 \leq A T<12 \\
& \text { ….... } 5 \\
& \boldsymbol{R F M}=1.3 *(\boldsymbol{A T T})^{1 / 3} \\
& A T \geq 12 \\
& 6
\end{aligned}
$$

The elastic cross section (shown in Figure 3 ) is obtained by taking the difference between the total and inelastic cross sections from ref.[8]. Fig 1 in ref.[9] shows that the correction for quasi-elastic scattering is small. A power law fit to the data in Figure 3 gives a determination of SIGL as,

$$
S I G L=0.0099 *(A T)^{0.91}
$$

(c) Examples:

Element--molybdenum (Mo).

$$
\begin{aligned}
& \mathrm{AT}=96 \\
& \mathrm{ZT}=42 \\
& \mathrm{RHO}=10.2 \\
& \mathrm{VIP}=442 . \\
& \mathrm{XO}=10.0 \\
& \mathrm{RALG}=0.98 \\
& \mathrm{RFM}=5.95 \\
& \mathrm{SIGL}=0.630
\end{aligned}
$$


Compound--beryllium oxide (BeO).

$$
\begin{aligned}
& \mathrm{AT}=(9 / 25) * 9+(16 / 25) * 16=13.5 \\
& \mathrm{ZT}=(9 / 25) * 4+(16 / 25) * 8=6.6 \\
& \mathrm{RHO}=2.85 \\
& \text { VIP }=92 . \\
& \text { X0 }=40.9 \\
& \text { RALG }=14.3 \\
& \mathrm{RFM}=3.10 \\
& \text { SIGL }=0.106
\end{aligned}
$$

Mixture--heavy concrete[10], (silicon $0.7 \%$, iron $62.9 \%$, calcium $3.3 \%$, oxygen $32.2 \%$ ).

$$
\begin{aligned}
& \mathrm{AT}=0.007 * 28+0.629 * 56+0.033 * 40+0.322 * 16=41.9 \\
& \mathrm{ZT}=0.007 * 14+0.629 * 26+0.033 * 20+0.322 * 8=19.7 \\
& \text { RHO }=4.27 \\
& \text { VIP }=226 \\
& \text { XO }=17.7 \\
& \text { RALG }=4.13 \\
& \text { RFM }=4.52 \\
& \text { SIGL }=0.296
\end{aligned}
$$

Mixture--ordinary concrete[10], (silicon $10.2 \%$, iron $1.6 \%$, calcium

$21.1 \%$, magnesium $6.5 \%$, carbon $7.3 \%$, and oxygen $51.5 \%$ ).

$\mathrm{AT}=22.9$

$\mathrm{ZT}=11.4$

$\mathrm{RHO}=2.4$

$\mathrm{VIP}=143$

$\mathrm{XO}=26.1$

RALG $=10.9$

$\mathrm{RFM}=3.69$

SIGL $=0.171$

Mixture--moist soil[10], (silicon $22.8 \%$, aluminum 5.5\%, iron 2.9\%, calcium $6.1 \%$, magnesium $2.1 \%$, carbon $3.3 \%$, and oxygen $55.0 \%$ ).

$\mathrm{AT}=21.6$

$\mathrm{ZT}=10.7$

$\mathrm{RHO}=2.25$

$\mathrm{VIP}=135$.

$\mathrm{XO}=27.6$

RALG $=12.3$

$\mathrm{RFM}=3.62$

SIGL $=0.162$ 


\section{Other Applications:}

CASIM has been benchmarked with measurements to a factor of 2 or 3 so that for most applications, the calculation of parameters as given in Section 2 will be adequate. However, other situations arise outside of CASIM where more accurate values are required. Such examples are multiple scattering, target length determinations, and particle production fluxes. This section gives guidelines to obtain more accurate values for the radiation length and the interaction length than what would be obtained by substituting ZT and AT into the approximate formulas.

For elements listed in the Particle Properties Data Booklet[8], ZT, AT, $\boldsymbol{X}_{0}$, and $\lambda_{\mathrm{I}}$ should be taken directly. For compounds and mixtures a more accurate value can be obtained by using TABLE 2 and the chemical compositions. Thus,

$$
\frac{1}{X_{0}}=\sum_{m=1}^{n} \frac{w_{m}}{X_{0}}
$$

For example the radiation length for mylar $\left(\mathrm{C}_{5} \mathrm{H}_{4} \mathrm{O}_{2}\right)$ would be,

$$
\begin{aligned}
& \frac{i^{*} A(C)+j * A(D)+k^{*} A(E)}{X_{0}(\text { mylar })}=\frac{i * A(C)}{X_{0}(C)}+\frac{j * A(D)}{X_{0}(D)}+\frac{k^{*} A(E)}{X_{0}(E)} \\
& \frac{96}{X_{0}(\text { mylar })}=\frac{5 * 12}{42.6983}+\frac{4 * 1}{63.0470}+\frac{2 * 16}{34.2381} \\
& X_{0}(\text { mylar })=39.95 \mathrm{~g} / \mathrm{cm}^{2}
\end{aligned}
$$

For a mixture like air, assuming $76.9 \%$ nitrogen, $21.8 \%$ oxygen, and $1.3 \%$ argon,

$$
\begin{aligned}
& \frac{1}{X_{0}}=\frac{.769}{37.9879}+\frac{.218}{34.2381}+\frac{.013}{19.5489} \\
& X_{0}=36.66 \mathrm{~g} / \mathrm{cm}^{2}
\end{aligned}
$$

Note that for these compounds/mixtures, the calculated values agree with those given in ref.[8].

At present, CASIM calculates the interaction length, $\lambda_{1}$ by taking the atomic weight (AT) and interpolating from four materials--beryllium, aluminum, copper, and lead-- $(9,85),.(27,113),.(64,141$.$) and (208,197$.$) .$ (Note for historical purposes, that the CASIM printout calls $\lambda_{\mathrm{I}}$ "Collision Length for Nucleons." The CASIM "collision length" is the "nuclear interaction length" given in ref. [8] modified by incoherent elastic scattering from the individual nucleons in the nuclei (quasi-elastic scattering)). The 
same method can be applied to find $\lambda_{\mathrm{I}}$ for compounds and mixtures by substituting $\lambda_{\mathrm{I}}$ for $\boldsymbol{x}_{0}$ in equation 8 .

$X_{0}$ and $\lambda_{\mathrm{I}}$ for the elements are listed in TABLE 2 in the columns X0 and L. Both are in units of $\mathrm{gm} / \mathrm{cm}^{2} . X_{0}$ is taken from Tsai[5], as are ZT and AT. $\lambda_{1}$ comes from two sources: $(1)$ the Particle Properties Data Booklet[8] when available, and (2) a supplemental calculation $\mathrm{AT} /\left(\mathrm{N}^{\star} \mathrm{CS}\right)$. $\mathbf{N}$ is Avogadro's number and $\mathbf{C S}$ is the inelastic cross section which was parameterized by Carroll[9] as $\operatorname{CS}($ barn $)=0.038^{\star} \mathrm{AT}^{\star \star} 0.72$.

\section{References}

[1] A. Van Ginneken, "CASIM--Program to Simulate Transport of Hadronic Cascades in Bulk Matter." (Batavia, IL.: Fermilab Internal Report FN-272, 1975).

[2] E. Segre, Nuclei and Particles, 2nd ed., (Reading, Massachusetts: The Benjamin/Cummings Publishing Company, Inc., 1977), 31.

[3] U.S. Department of Health Education and Welfare, Radiological Health Handbook, (Washington, D. C.: U.S. Government Printing Office, 1970), 65.

[4] Particle Data Group, "Review of Particle Properties", Physical Review D45, Part II, (1992), III.15.

[5] Y. S. Tsai, "Pair production and bremsstrahlung of charged leptons", Reviews of Modern Physics 46 (1974), 815.

[6] G. Bellettini, et al., "Proton-Nuclei Cross Sections at $20 \mathrm{GeV}$ ", Nuclear Physics 79 (1966), 609.

[7] R. Herman and R. Hofstadter, High-Energy Electron Scattering Tables, (Stanford, CA: Stanford University Press, 1960), 61.

[8] Ibid., Physical Review D45, III.5.

[9] A. S. Carroll et al., "Absorption Cross Section of $\mathrm{Pi}^{ \pm}, \mathrm{Ka}^{ \pm}, \mathrm{p}$ and pbar on Nuclei Between 60 and $280 \mathrm{GeV} / \mathrm{c}^{\prime}$, Physics Letters, 80B (1979), 319.

[10] M. Awschalom, T. Borak, and P. Gollon, "Chemical Composition of Some Common Shielding Materials." (Batavia, IL.: Fermilab Internal Report TM-168, 1969), 3. 
TABLE 2: Radiation Length $\left(X_{0}\right)$ and Interaction Length(L) of the Elements

\begin{tabular}{|c|c|c|c|c|}
\hline ZT & AT & $\mathrm{XO}(\mathrm{g} / \mathrm{sg} . \mathrm{cm})$ & CS(barn) & $\mathrm{L}(\mathrm{g} / \mathrm{sq} \cdot \mathrm{cm})$ \\
\hline 1 & 1.008 & 63.047 & 0.033 & 50.7 \\
\hline 2 & 4.003 & 94.322 & 0.102 & 65.2 \\
\hline 3 & 6.939 & 82.756 & 0.157 & 73.4 \\
\hline 4 & 9.012 & 65.190 & 0.199 & 75.2 \\
\hline 5 & 10.811 & 52.687 & 0.213 & 84.4 \\
\hline 6 & 12.011 & 42.698 & 0.231 & 86.3 \\
\hline 7 & 14.007 & 37.988 & 0.265 & 87.8 \\
\hline 8 & 15.999 & 34.238 & 0.292 & 91.0 \\
\hline 9 & 18.998 & 32.930 & 0.319 & 98.9 \\
\hline 10 & 20.183 & 28.937 & 0.347 & 96.6 \\
\hline 11 & 22.990 & 27.736 & 0.366 & 104.4 \\
\hline 12 & 24.312 & 25.039 & 0.381 & 106.0 \\
\hline 13 & 26.982 & 24.011 & 0.421 & 106.4 \\
\hline 14 & 28.086 & 21.823 & 0.440 & 106.0 \\
\hline 15 & 30.974 & 21.205 & 0.453 & 113.5 \\
\hline 16 & 32.064 & 19.495 & 0.465 & 114.6 \\
\hline 17 & 35.453 & 19.278 & 0.499 & 117.9 \\
\hline 18 & 39.948 & 19.549 & 0.566 & 117.2 \\
\hline 19 & 39.102 & 17.317 & 0.536 & 121.2 \\
\hline 20 & 40.080 & 16.144 & 0.546 & 122.0 \\
\hline 21 & 44.956 & 16.546 & 0.592 & 126.0 \\
\hline 22 & 47.900 & 16.174 & 0.637 & 124.9 \\
\hline 23 & 50.942 & 15.842 & 0.648 & 130.5 \\
\hline 24 & 51.996 & 14.944 & 0.658 & 131.3 \\
\hline 25 & 54.938 & 14.640 & 0.684 & 133.3 \\
\hline 26 & 55.847 & 13.839 & 0.703 & 131.9 \\
\hline 27 & 58.933 & 13.617 & 0.720 & 136.0 \\
\hline 28 & 58.710 & 12.682 & 0.718 & 135.8 \\
\hline 29 & 63.540 & 12.862 & 0.782 & 134.9 \\
\hline 30 & 65.370 & 12.427 & 0.776 & 140.0 \\
\hline 31 & 69.720 & 12.473 & 0.812 & 142.5 \\
\hline 32 & 72.590 & 12.246 & 0.858 & 140.5 \\
\hline 33 & 74.922 & 11.940 & 0.855 & 145.4 \\
\hline 34 & 78.960 & 11.908 & 0.888 & 147.6 \\
\hline 35 & 79.909 & 11.423 & 0.896 & 148.1 \\
\hline 36 & 83.800 & 11.372 & 0.927 & 150.1 \\
\hline 37 & 85.470 & 11.027 & 0.940 & 150.9 \\
\hline 38 & 87.620 & 10.762 & 0.957 & 152.0 \\
\hline 39 & 88.905 & 10.410 & 0.967 & 152.6 \\
\hline 40 & 91.220 & 10.195 & 0.985 & 153.7 \\
\hline 41 & 92.906 & 9.922 & 0.999 & 154.5 \\
\hline 42 & 95.940 & 9.803 & 1.022 & 155.9 \\
\hline 43 & 99.000 & 9.688 & 1.045 & 157.3 \\
\hline 44 & 101.070 & 9.482 & 1.061 & 158.2 \\
\hline 45 & 102.905 & 9.265 & 1.075 & 159.0 \\
\hline 46 & 106.400 & 9.202 & 1.101 & 160.5 \\
\hline
\end{tabular}


TABLE 2: Radiation Length $\left(X_{0}\right)$ and Interaction Length $(L)$ of the Elements

\begin{tabular}{|c|c|c|c|c|}
\hline $\mathbf{Z T}$ & AT & $\mathrm{XO}(\mathrm{g} / \mathrm{sq} . \mathrm{cm})$ & CS(barn) & $\mathrm{L}(\mathrm{g} / \mathrm{sq} . \mathrm{cm})$ \\
\hline & & & & \\
\hline 47 & 107.870 & 8.970 & 1.112 & 161.1 \\
\hline 48 & 122.400 & 8.994 & 1.217 & 167.0 \\
\hline 49 & 114.820 & 8.849 & 1.163 & 164.0 \\
\hline 50 & 118.690 & 8.817 & 1.210 & 162.9 \\
\hline 51 & 121.750 & 8.724 & 1.213 & 166.7 \\
\hline 52 & 127.600 & 8.827 & 1.254 & 168.9 \\
\hline 53 & 126.904 & 8.480 & 1.249 & 168.7 \\
\hline 54 & 131.300 & 8.482 & 1.290 & 169.0 \\
\hline 55 & 132.905 & 8.305 & 1.292 & 170.9 \\
\hline 56 & 137.340 & 8.307 & 1.323 & 172.4 \\
\hline 57 & 138.910 & 8.138 & 1.333 & 173.0 \\
\hline 58 & 140.120 & 7.956 & 1.342 & 173.4 \\
\hline 59 & 140.907 & 7.758 & 1.347 & 173.7 \\
\hline 60 & 144.240 & 7.705 & 1.370 & 174.8 \\
\hline 61 & 145.000 & 7.519 & 1.375 & 175.1 \\
\hline 62 & 150.350 & 7.573 & 1.411 & 176.9 \\
\hline 63 & 151.960 & 7.438 & 1.422 & 177.4 \\
\hline 64 & 157.250 & 7.483 & 1.458 & 179.1 \\
\hline 65 & 158.924 & 7.356 & 1.469 & 179.7 \\
\hline 66 & 162.500 & 7.320 & 1.493 & 180.8 \\
\hline 67 & 164.930 & 7.233 & 1.509 & 181.5 \\
\hline 68 & 167.260 & 7.145 & 1.524 & 182.3 \\
\hline 69 & 168.934 & 7.032 & 1.535 & 182.8 \\
\hline 70 & 173.040 & 7.021 & 1.562 & 184.0 \\
\hline 71 & 174.970 & 6.924 & 1.574 & 184.6 \\
\hline 72 & 178.490 & 6.891 & 1.597 & 185.6 \\
\hline 73 & 180.948 & 6.818 & 1.613 & 186.3 \\
\hline 74 & 183.850 & 6.763 & 1.650 & 185.0 \\
\hline 75 & 186.200 & 6.690 & 1.646 & 187.8 \\
\hline 76 & 190.200 & 6.676 & 1.671 & 189.0 \\
\hline 77 & 192.200 & 6.594 & 1.684 & 189.5 \\
\hline 78 & 195.090 & 6.543 & 1.708 & 189.7 \\
\hline 79 & 196.967 & 6.461 & 1.714 & 190.8 \\
\hline 80 & 200.590 & 6.437 & 1.737 & 191.8 \\
\hline 81 & 204.370 & 6.418 & 1.760 & 192.8 \\
\hline 82 & 207.190 & 6.369 & 1.770 & 194.4 \\
\hline 83 & 208.980 & 6.290 & 1.788 & 194.0 \\
\hline 84 & 210.000 & 6.191 & 1.795 & 194.3 \\
\hline 85 & 210.000 & 6.065 & 1.795 & 194.3 \\
\hline 86 & 222.000 & 6.283 & 1.868 & 197.4 \\
\hline 87 & 223.000 & 6.187 & 1.874 & 197.6 \\
\hline 88 & 226.000 & 6.148 & 1.892 & 198.4 \\
\hline 89 & 227.000 & 6.056 & 1.898 & 198.6 \\
\hline 90 & 232.038 & 6.073 & 1.928 & 199.8 \\
\hline 91 & 231.000 & 5.932 & 1.922 & 199.6 \\
\hline 92 & 238.030 & 5.999 & 1.980 & 199.6 \\
\hline
\end{tabular}




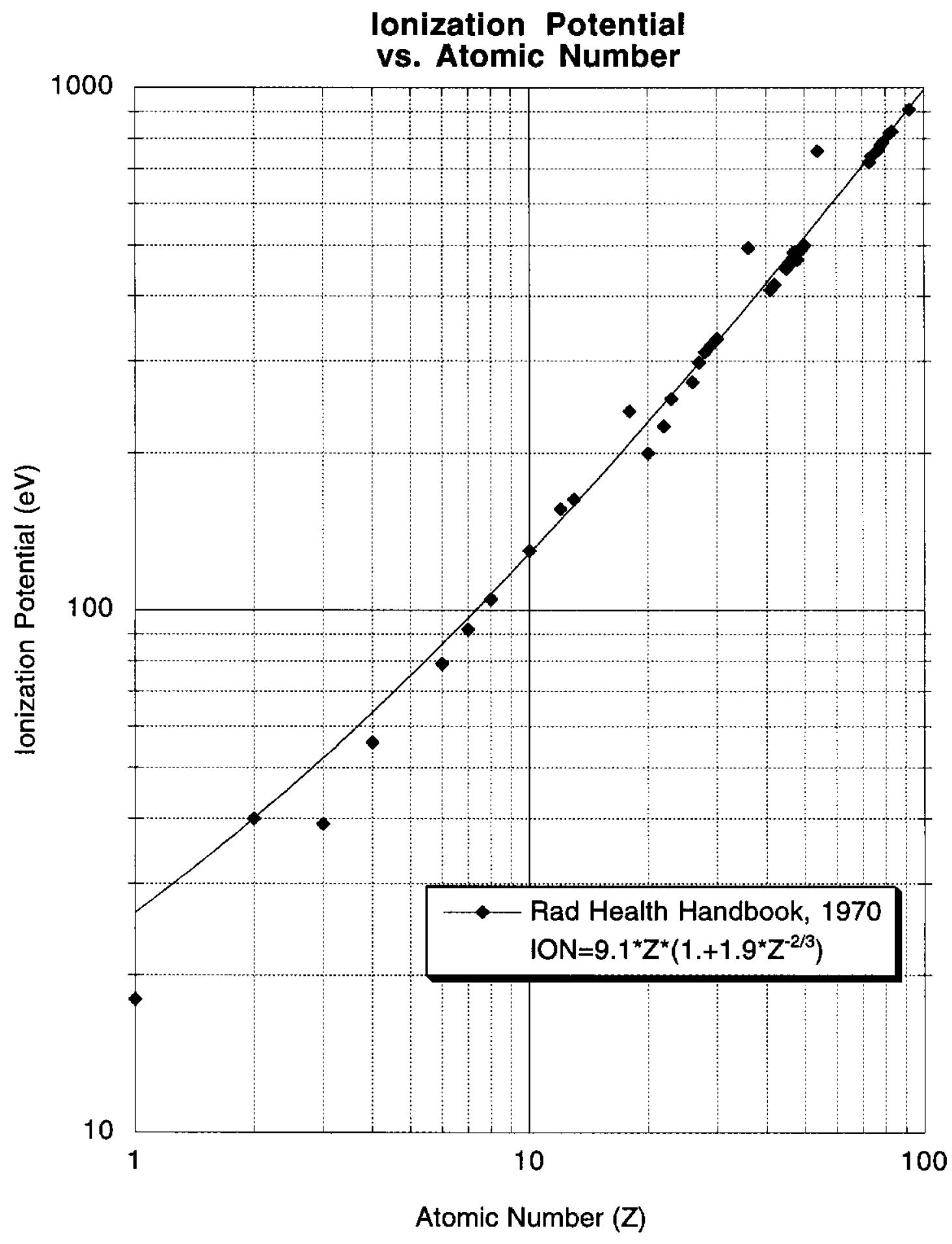

Figure 1 


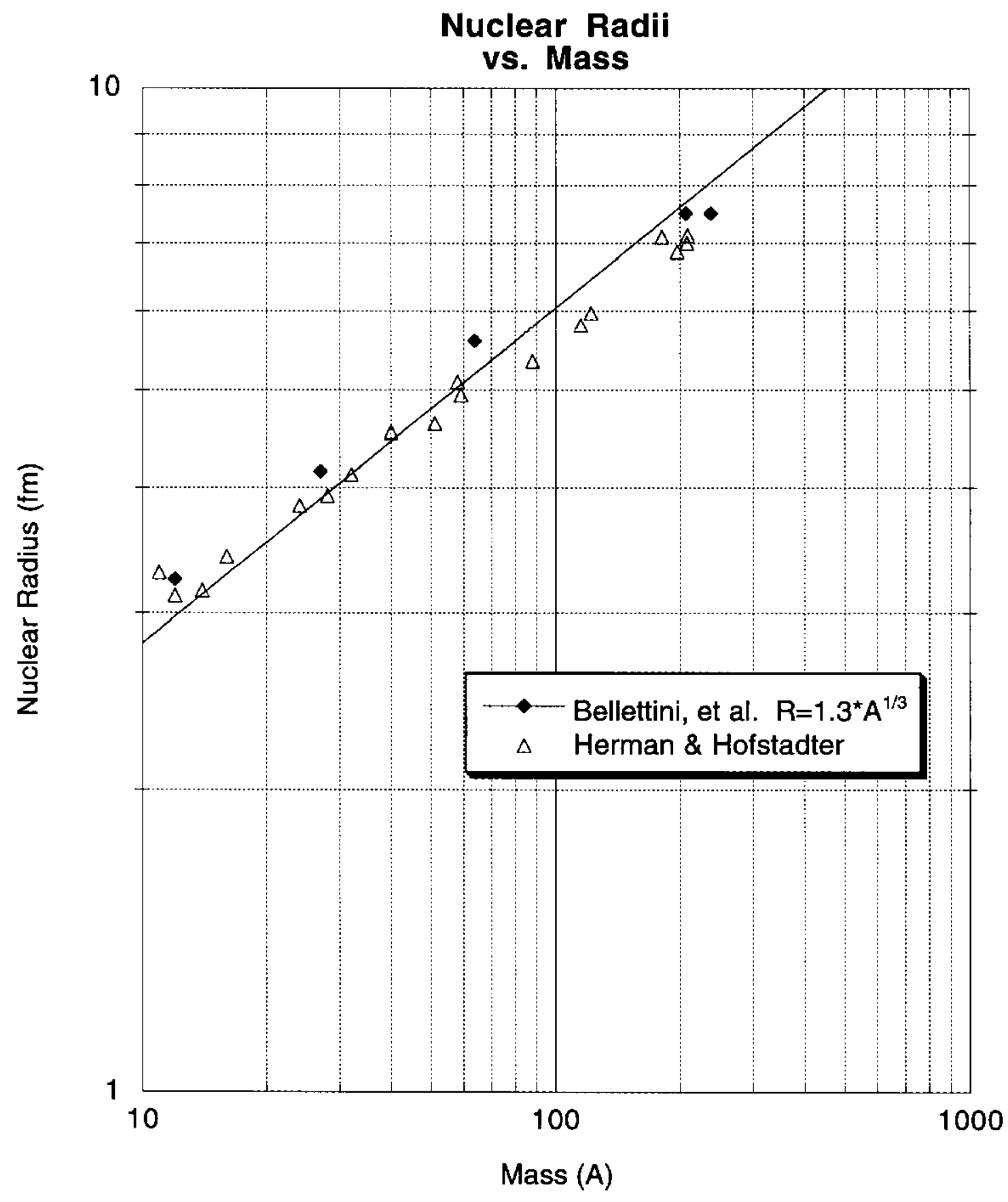

Figure 2 


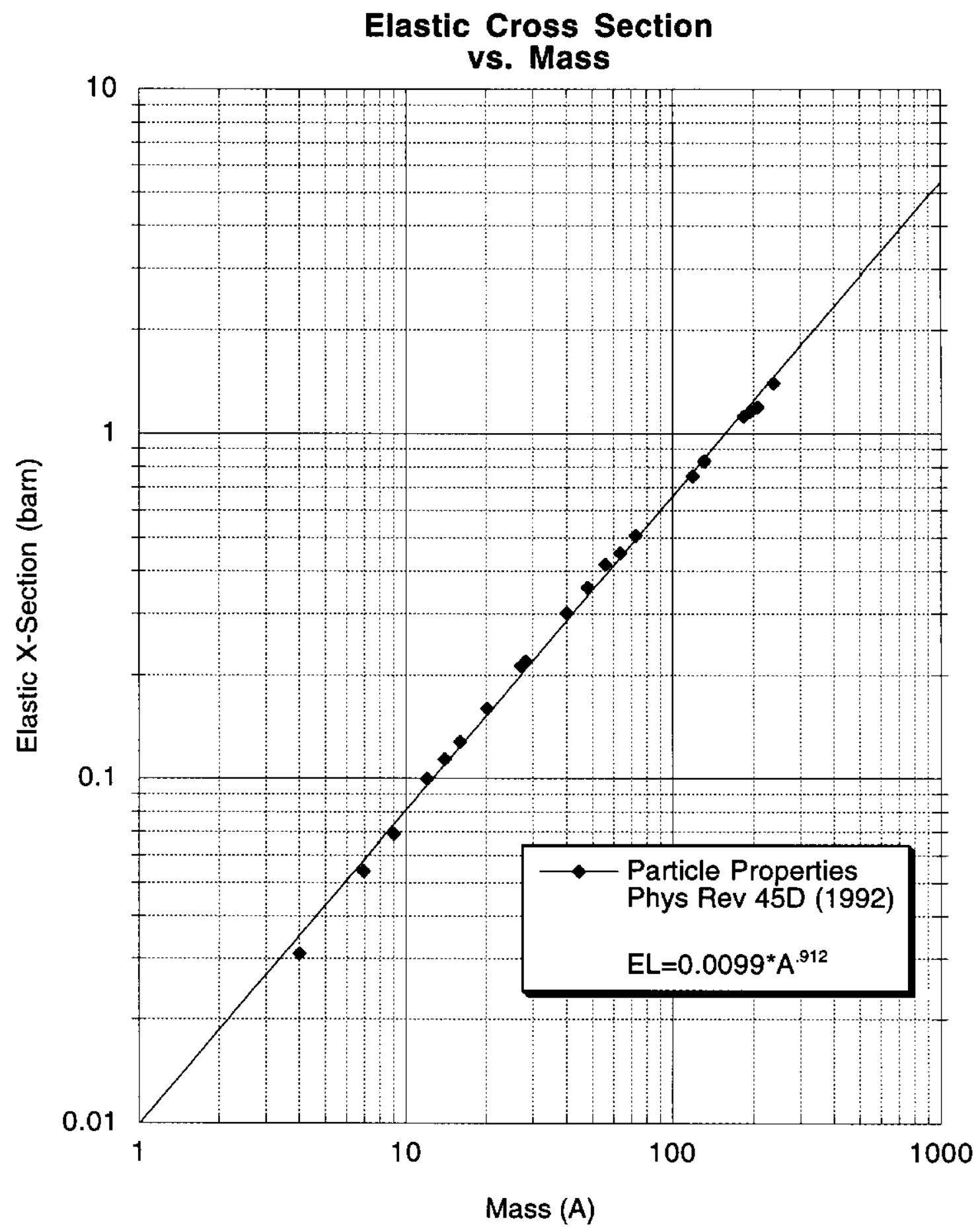

Figure 3 